\title{
YUKIO TOMOZAWA
}

Randall Lab of Physics, University of Michigan, Ann Arbor, MI 48109-1120 ABSTRACT

The suggestion has been made that the energy spectrum from point sources such as AGN (Active Galactic Nuclei) and GBHC (Galactic Black Hole Candidates) is universal, irrespective of the nature of the emitted particles. A comparison of the energy spectrum for cosmic rays at the source and $\gamma$-rays from quasars obtained recently by CGRO (Compton Gamma Ray Observatory) indicates that the prediction is in agreement with the data in the average sense. This suggests that neutrinos from point sources should have a spectral index identical to that of $\gamma$-rays for an individual point source. This prediction is also consistent with the recent obervation of neutrinos by Kamiokande and IMB in which the ratio of $\nu_{\mu} / \nu_{e}$ is close to 1 , instead of 2 as expected from atmospheric neutrinos. For a further test of the model, analysis of the time variation of $\gamma$-ray spectra from quasars is suggested.

\section{Introduction}

The observation of high energy $\gamma$-rays from quasars (up to a few $\mathrm{GeV}$ by EGRET of CGRO ${ }^{[1,2]}$ and up to a few TeV by the Whipple Observatory ${ }^{[3]}$ clearly calls for a drastically new approach for understanding the $\gamma$-ray emission mechanism from point sources. This is because electron infall onto a compact object generates $\mathrm{X}$-rays but not high energy $\gamma$-rays: (for such a process, (the gravitational energy) $/($ the rest energy) $\lesssim 1$ ). The purpose of this article is to present a novel mechanism of $\gamma$-ray emission from black holes and to compare its prediction with the observed data. In fact, the model ${ }^{[4-8]}$ was proposed in 1985, much before the CGRO operation, and the recent data is quite consistent with the prediction.

The salient features of the theory can be summarized in the following way.

1. The consideration of quantum effects on the Einstein equation suggests that the gravitational potential is repulsive at short distances. The rotation of black holes represented by the Kerr metric has the similar feature, i.e. the angular momentum plays the role of a repulsive force, a phenomenon similar to the angular momentum barrier in quantum mechanics.

2. Applying this result to gravitational collapse, one encounters a novel phenomenon called black hole pulsation. An analysis of the behavior of black hole motion enables us to conclude that the pulsation is observable.

3. The spectrum of particles emitted during the pulsation is decided by the rate of expansion of the system. This leads to the prediction of a universal energy spectrum for different particles from an individual pulsating 
compact object. (There should be a variation for the spectrum since an individual object can have a different expansion rate depending on the environment.)

4. This prediction of universality can be compared with the data of cosmic rays, $\gamma$-rays and neutrinos. It will be shown that the data is in reasonable agreement with the prediction and further tests will be suggested.

Section II is devoted to a discussion of cosmic rays generated by pulsating black holes, and $\gamma$-rays from point sources observed by CGRO compared with the cosmic ray energy spectrum at the sources in Section III. Section IV presents a discussion on the neutrino observation by the Kamiokande and IMB detectors, which may provide evidence for the proposed model and Section $V$ suggests the analysis of time variation of the energy spectrum as a further test of the theory.

\section{Cosmic Rays}

Since the discovery of cosmic rays early this century, an impressive amount of experimental data has been accumulated. Yet, the origin of cosmic rays defies the understanding of physicists. Several important questions are: What is the fraction of galactic and extragalactic components of primary cosmic rays? How can one understand the power law energy spectrum $\left(\sim E^{-2.5}-E^{-3}\right.$ for the energy range $10^{9} \mathrm{eV}<E<10^{20} \mathrm{eV}$ )? How do they attain such high energies? Despite various attempts in the past to answer these questions, we are still left in the dark. ${ }^{[7]}$

It is known ${ }^{[8]}$ that shock wave acceleration in a supernova explosion does not explain the high energy component of cosmic rays (above the so called knee energy $10^{16} \mathrm{eV}$ ). It was then suggested that strong magnetic fields around pulsars may be responsible for the acceleration of high energy cosmic rays ${ }^{[\theta]}$ above the knee energy. In this case, however, drastically different mechanisms are responsible for cosmic ray acceleration below and above the knee energy. This makes it difficult to have the continuous spectrum observed in the experimental data. An alternative scheme is to invoke shock wave acceleration in the galactic wind, ${ }^{[10]}$ the existence of which is yet to be established by observation.

The application of BHP (black hole pulsation) leads naturally to the emission of particles. Since the temperature decreases with expansion of quantum mechanical black holes or rotating black holes, the energy spectrum of the emitted particles can be computed, provided the expansion rate is known.

The number of particles of type $x$ emitted with energy $E$ is given by

$$
f_{x}(E)=\frac{(2 s+1)}{2 \pi^{2}} \int \eta_{x}(E / k T) \frac{E^{2} 4 \pi R^{2} d t}{e^{E / k T-\mu / k T} \pm 1},
$$

where $R$ is the radius of the system, $\eta_{x}(E / k T) d t$ is the fraction of particles $x$ emitted in time interval $d t$ and $\mu$ and $s$ are the chemical potential and the 
spin for particles of type $x$. The $+(-)$ sign in the denominator is for fermions (bosons). Assume the relationship $R=a / k T$ and an expanding rate

$$
t=b R^{\alpha}
$$

where $a$ and $b$ are constants. The function $\eta_{x}(E / k T)$ is unknown, but is assumed to scale as a function of $E / k T$. The chemical potential for fermions is obtained by the condition

$$
\frac{N}{V}=\frac{(2 s+1)}{2 \pi^{2}}(k T)^{3} \int_{0}^{\infty} \frac{x^{2} d x}{e^{x-\mu / k T}+1}
$$

or equivalently

$$
\mu_{0} \equiv \mu / k T=g\left(\frac{N}{V(k T)^{3}} \frac{2 \pi^{2}}{(2 s+1)}\right),
$$

where $N$ is the total number of particles in the system and $V$ is the volume given by

$$
V=\frac{4 \pi}{3} R^{3}=\frac{4 \pi}{3} \frac{a^{3}}{(k T)^{3}} .
$$

(At high temperature $g(x)=\ell n(x / 2)$.) Obviously, $\mu_{0}$ is independent of temperature since $V T^{3}$ is constant during the course of the expansion. $\left(\mu_{0}=0\right.$ for photons.) Using Eqs. (1)-(4), we obtain

$$
f_{x}(e)=\frac{A_{x, \alpha}}{E^{\alpha}}
$$

where

$$
A_{x, \alpha}=\frac{2(2 s+1) \alpha b(a)^{2+\alpha}}{\pi} \int_{0}^{\infty} \frac{\eta_{x}(s) s^{\alpha+1} d s}{e^{s-\mu_{0}} \pm 1}
$$

is a constant.

Some discussion is in order. First, how can one explain the observed nuclei in cosmic rays $(\sim 10 \%$ of primary cosmic rays which are mostly protons below the knee energy). One can invoke shock wave acceleration in supernova explosions for $10-20 \%$ of cosmic rays. Or nuclei can be emitted from BHP, since the density is extremely high so that the Fermi temperature is also extremely high. As a result, the situation can be like a low temperature state despite the temperature $T$ is high. Thus, due to the Boson condensation, even nuclei can be emitted in this system. ${ }^{[11]}$ 
Secondly, the cosmic ray energy spectrum observed at the earth may not be the same as that at the sources. Using leakage, spallation and information on the chemical abundance of cosmic rays, the Chicago group has derived the power index $\lambda$ of the cosmic ray energy spectrum $E^{-\lambda}$ at the sources. The most elaborate analysis ${ }^{[12]}$ gives

$$
\lambda_{\text {source }}=2.2 \pm 0.1 \text {. }
$$

This index should be compared with the index for $\gamma$-ray energy spectrum.

Finally, in our model the power law spectrum of cosmic rays is a reflection of the power law expansion rate. The knee energy is caused by the difference of the expansion rate in the nonrelativistic and relativistic regime. Then, this would require the existence of the energy scale of $\sim$ several hundred $\mathrm{TeV}$ which differentiates both regimes. However, the modification of the low energy spectrum with Eq. (8) brings the knee energy at a much lower energy scale, around $1 \mathrm{TeV}$ (instead of several hundred $\mathrm{TeV}$ ).

\section{Gamma-Rays}

It is clear that the energy spectrum derived in the last section applies for any particles emitted from BHP. The important prediction is, then

[Proposition] Any particles emitted from point sources such as GBHC or AGN should have identical energy spectrums on the average, the universal spectrum, when they left the sources.

The most recent data by the EGRET detector gives the energy spectrum for $\gamma$-rays from quasar $3 \mathrm{C} 279$ as $E^{-\lambda}$, where ${ }^{[1]}$

$$
\lambda=2.02 \pm 0,07,
$$

for the energy range $30 \mathrm{MeV}$ to $5 \mathrm{GeV}$. The same group observed ${ }^{[2]}$ three bursts from the location at R.A. $=88.6$ degrees and Dec. $=38.6$ degrees. They have power index

$$
\lambda=2.13 \pm 0.08,
$$

and

$$
\lambda=2.24 \pm 0.03 .
$$

The spectrum of the third burst is more complicated and it is suggested as a composite of

$$
\lambda=2.22,
$$

and

$$
\lambda=4.0 \pm 0.8 \text { at the low energy end. }
$$

The proximity of the values of Eqs. (8), (9) and (10) seems to support the concept of universality proposed by the author. It should be emphasize, however, 


\section{Neutrinos from Point Sources}

that universality of the energy spectrum is valid only in the sense of the average so that a certain amount of fluctuation is inevitable. As a matter of fact, the spectrum of cosmic rays itself should be the outcome of an average of the spectrum from many sources which power index has a fluctuation.

\section{Muon Neutrino Deficiency and Cosmic Neutrinos}

The concept of a universal energy spectrum from point sources can be applied to other particle emissions. An example is neutrinos. Neutrinos are emitted from pulsating black holes with an intensity comparable to that of $\gamma$-rays. With the difference of statistics and helicity, the neutrino spectrum is $3 / 8$ of the $\gamma$-ray spectrum, but they have the same spectral index (with the same variation for individual sources, of course). Moreover, the intensity is the same for all species $\nu_{e}, \bar{\nu}_{e}, \nu_{\mu}, \bar{\nu}_{\mu}, \nu_{\tau}$ and $\bar{\nu}_{\tau}$ and they have the advantage that their flux is hardly modified once it leaves the sources.

Recently, the underground neutrino detectors at Kamiokande II and IMB compiled neutrino flux between $100 \mathrm{MeV}$ and $1.5 \mathrm{GeV}$ and concluded ${ }^{[13]}$ that the ratio of $\nu_{\mu}$ and $\nu_{e}$ is 1 instead of 2 . The latter value of 2 is expected if the observed neutrinos are produced in the atmosphere, since pions and kaons are the neutrino source. This riddle, called the muon neutrino deficiency problem, is solved if neutrino oscillation $\left(\nu_{\mu} \rightarrow \nu_{x}\right)$ ensued after production in the atmosphere. ${ }^{[1,15]}$ However, study of the up-going nuon suggests that such oscillation does not take place. ${ }^{[15]}$ Also, it can be shown that the calculated atmospheric neutrino flux ${ }^{[16]}$ tends to be an overestimate. From these considerations, it is very likely that the neutrinos observed by the underground detectors are not atmospheric but cosmic, i.e. most neutrinos observed are coming from outside the atmosphere of the earth. But, of course, an ordinary mechanism for neutrino production ends up with $\nu_{\mu} / \nu_{e} \simeq 2$, by the same reason as for atmospheric neutrinos. The model proposed in this project is the only one which predicts $\nu_{\mu} / \nu_{e}=1$. Moreover, it si worthwhile to mention that the neutrino flux spectrum inferred from the underground detector data ${ }^{[13]}$ is close to $E^{-2.2}$. Further observation of the neutrino flux will decide the validity of the model.

\section{Summary and Further Predictions}

The prediction of universality for the energy spectrum from point sources (with variation of the spectral index for individual sources) is dramatically borne out by the GRO data and the cosmic energy spectrum at the source. Also an approximate equality of $\nu_{\mu}$ and $\nu_{e}$ in the underground detector lends support to this model.

In order to further confront the theory with observational data, I propose to analyze the time variation of the $\gamma$-ray spectrum. According to the model suggested in this article, the instantaneous spectrum of $\gamma$-rays from point sources is Planckian. Only after integration over the various temperature, can one get a power law spectrum as a reflection of a power law expansion rate. For example, 
the variability of $\mathrm{X}$-rays from quasar $3 \mathrm{C} 273$ is $\sim 2$ days. Therefore, we need a slicing of the data in the time bin less than 2 days for $3 \mathrm{C} 273$. Of course, the slicing of data into small time intervals results in the data of poor statistics. Therefore, an appropriate integration of the data may be necessary. In conclusion, I propose to analyze the CGRO data and extract information on the time variation of the spectrum.

The author is indebted to David Williams for reading the manuscript. The work is supported in part by the U.S. Department of Energy.

\section{REFERENCES}

1. R.C. Hartman et al., Ap. J. $\underline{385}$ L1 (1992).

2. E.J. Schneid et al., Astron. Astrophys., to appear.

3. M. Shubnell, Proc. Seventh International Symposium on Very High Energy Cosmic Ray Interactions; Nature in press.

4. Y. Tomozawa, Proceedings of the INS International Symposium on Composite Models of Quarks and Leptons (ed. H. Terazawa and M. Yasue, 1985), p. 386.

5. Y. Tomozawa, in Quantum Field Theory (ed. F. Mancini, Elsevier Pub., 1986), p. 241.

6. Y. Tomozawa, Lectures at the Second Workshop on Fundamental Physics, Univ. of Puerto Rico, Humacao (ed. E. Estaban, 1986), p. 144.

7. M.S. Longair, High Energy Astrophysics (Cambridge University Press, 1981); S. Hayakawa, Cosmic Ray Physics (Wiley-Interscience, NY, 1969).

8. A.R. Bell, M.N.R.S., 182 , 147, 443 (1978); P.O. Lagage and C.J. Casarsky, Astr. Ap. 118,223 (1983); R.D. Blandford and J.P. Ostriker, Ap. J. 221, L29 (1978).

9. J.E. Gunn and J.P. Ostriker, Phys. Rev. Lett. 22, 728 (1969).

10. J.P. Jokipii and G.E. Morfill, Ap. J. 290 , L1 (1985).

11. A. Majumder and Y. Tomozawa, Prog. Theoret. Phys. 82,555 (1989).

12. S.P. Swordy et al., Ap. J. $\underline{349}$ (1990) 625; D. Müller et al., Ap. J. $\underline{374}$ (1991) 356; P. Meyer et al., ICRC (1991) OG6.1.11.

13. K.S. Hirata et al., Phys. Lett. B280, 146 (1992).

14. E.W. Beier et al., Phys. Lett. B283, 440 (1992).

15. R. Becker-Szendy et al., Phys. Rev. Letters, to appear (1992).

16. e.g., E.V. Bugaev and V.A. Naumov, 20th International Cosmic Ray Conference, HE 4.1-18 (1987); Sov. J. Nucl. Phys. 45, 857 (1987); G. Barr, T.K. Gaisser and T. Stanev, Phys. Rev. D39, 3532 (1989). 